\title{
Polymeric Micelles for Cancer Drug Delivery and Targeting
}

\author{
Yuyan Wang ${ }^{1, *}$ \\ ${ }^{1}$ University of California Irvine, Physical Department, 92697, Irvine, United States
}

\begin{abstract}
Over the past years, the emergence of various therapeutic approaches has attracted so much consideration, but chemotherapy remains the most effective way in experimental practice. However, due to obstacles and difficulties, there is a limitation for the additional clinical presentation of the outdated chemical treatments such as paclitaxel and doxorubicin. Some of the barriers are severe side effects, profound cytotoxicity, and low therapeutic efficacy. Drug delivery systems (DDS) such as polymeric micelles have been technologically advanced over the recent decades to advance the treatment efficacy and lessen the side effects incurred. The polymeric micelles self-assembled from amphiphilic polymers serve to sum up hydrophobic treatments in the core and regulate the discharge profile of the drugs through response towards stimuli of the tumor microenvironments, which in this case are $\mathrm{pH}$, temperature, light, and reduction.
\end{abstract}

\section{Introduction}

Recently, polymer micelles techniques have pulled in expanded consideration as a good vehicle for inadequately dissolvable medications in modern cancer treatment [1]. Polymer micelles are self-congregations of amphiphilic block copolymers in aqueous media. Meanwhile, many benefits of utilizing polymer micelles have been exhibited with their unique core-shell structure. The hydrophobic centres are isolated by hydrophilic shells from the aqueous exterior, so then hydrophobic medications can be solubilized into the hydrophobic centre designs of polymer micelles at focuses a lot higher than their characteristic water-solvency [2]. Polymer micelles have a high medication stacking limit, high water-dissolvability, and fitting size for a long course in blood. The hydrophilic shell encompassing the micellar centre can secure unfortunate marvels, for example, between micellar conglomeration or precipitation, protein adsorption, and cell bond. The substance organization of polymer micelles can be customized to have alluring strong compound properties for the drug solubilization. In most polymer micelles, hydrophobic medications are joined into the hydrophobic centre of micelles by hydrophobic collaboration just as other extra communications, for example, metal-ligand coordination holding an electrostatic connection. The drug solubility's ability depends on the compatibility of drug and the micelle core. This study focus on the structure of polymeric micelle, the procedure of polymeric micelle formation, and the methods used to stimulated the drug delivery, which included $\mathrm{pH}$-sensitive, photosensitive, thermal-sensitive, and reduction.

\subsection{Overview of self-assembly}

\subsubsection{Structure}

Polymeric micelle has a small size, and usually their diameter ranges from 5 to $100 \mathrm{~nm}$ [3], which depends on the type of head and length of function groups. Polymeric micelles are based on block-copolymers with hydrophilic and hydrophobic units that self-assemble in an aqueous environment into structures These blocks can be arranged in different ways: A-B type, A-B-A type [4]. In the micelle structure formation process, the hydrophobic and hydrophilic are separated due to the interaction between the polar part of the head and surrounding water [5]. This formation process results in a flexible and porous structure of micelle. Now, polymer micelle is widely used as a high effectivity model of biological application and drug delivery systems because of the special function polymer micelle. For example, they can increase the drug solubility from the targeting ability, reduce the toxicity, and enhance the penetration on tissue. Polymeric micelles are composed of the amphiphilic molecules in an aqueous solution, then self-assemble into a core-shell structure that contains both hydrophobic and hydrophilic segments.

For the solution, the critical concentration required in forming polymeric micelle is called critical micelle concentration (CMC). The formation of micelle occurred when $\mathrm{CMC}$ value higher than the concentration needed [6]. The typical micelle formation is driven by dehydration of the hydrophobic tails. The final structure of polymeric micelles are spherical, supramolecular molecule, and micelle have a unique morphologies ability can be assembled in different sharp, for example, rods, spheres, and tubules, which depend on the temperature of the environment, chain's length, and quality of solvent. Polymeric micelle's function is not limited to cancer

\footnotetext{
* Corresponding author: yuyanw7@uci.edu
} 
therapy, but also in different fields, such as material engineers and microelectronics.

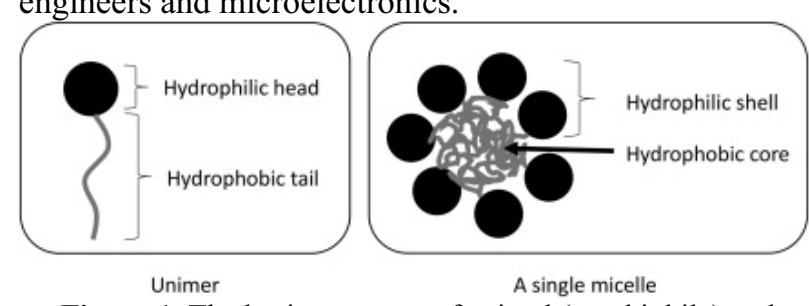

Figure 1. The basic structure of animal (amphiphile) and combine polymeric micelle [7].
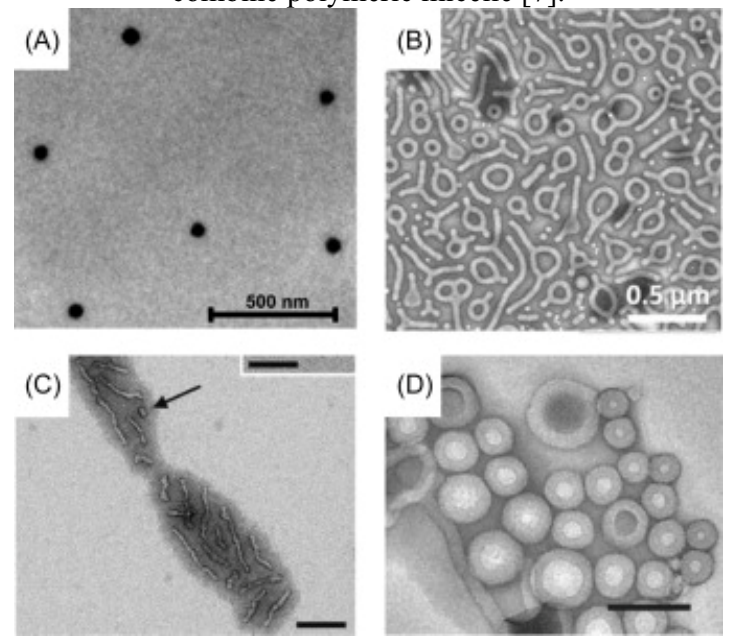

Figure 2. The micelle morphologies under transmission electron micrographs: (A) spheres, (B) toroids, (C) worm, (D) vesicles.

\subsection{Synthesis of polymer}

The synthesis of polymers has been a great interest and popular field in polymer chemistry. Among those polymer technologies, living polymerization techniques are widely used, as it can employ in the polymerization proceed under a lack of irreversible chain transfer and chain termination condition. Recently, cutting-edge technology called controlled/living radical polymerization (CRP) has been developed, as the radical process is more tolerant of functional groups and impurities. The rapid dynamic equilibration was the foundation of the new CRP methods, between a small amount of growing free radicals and many dormant species.

\subsubsection{ATRP}

One method focuses on transition metal-catalyzed atom transfer radical polymerization (ATRP). ATRP was based on the atom transfer step, which is a key element in the uniform growth of the polymeric chains [8]. ATRP was target to form 1:1 adducts of alkyl halides and alkenes, and its originated atom transfer radical addition (ATRA) $[9,10]$. ATRA is one kind of Kharasch additional reaction under the presence of light or conventional radical initiators. The ATRP mechanism is controlled by the equilibrium between propagation radicals and dormant functional groups, mainly in the form of initiating alkyl halides or macromolecular species. For the dormant species, they can react with the rate constant of activation ( $\mathrm{k}_{\text {eact }}$ ) with a transition metal, for example, copper, in the lower oxidation state. During the reaction, polymer chains grow in the constant rate of propagation $\mathrm{k}_{\mathrm{p}}$ by adding the intermediate radicals to monomers in the solution, so the chains growth process is similar to conventional radical polymerization. In addition, termination reactions $\left(\mathrm{k}_{\mathrm{t}}\right)$ are also shown in ATRP reaction, in the radical coupling and disproportionation process. However, only a meager percentage of polymeric chains pass the termination section. Less than $5 \%$ of the total growing polymer chains end the reaction in the lab during the initial stage of the polymerization. This process results in the growing radicals $(\mathrm{Pn} \bullet)$ and formed transition metal complexes (X$\mathrm{M}_{\mathrm{t}}^{\mathrm{n}+1}$ ) in a higher oxidation state. The oxidized metal complexes can minimize the influence of termination by reducing the concertation of growing radicals. Under the reverse reaction $\left(\mathrm{k}_{\text {deact }}\right)$ condition, propagating radicals can reform the dormant species and the activator.

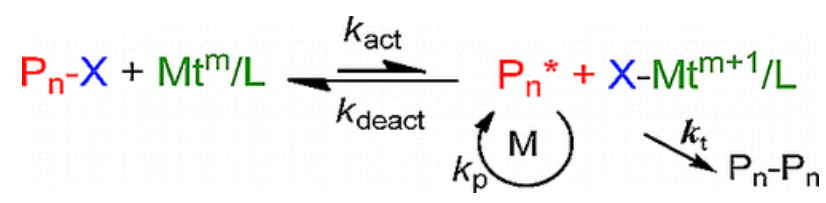

Figure 3. ATRP equilibrium mechanism.

ATRP is a catalysis reaction, and $\mathrm{CuI} / \mathrm{L}$ and $\mathrm{X}-\mathrm{CuII} / \mathrm{L}$ are the most commonly used transition metals in response. Its rate is based on the rate constant of propagation, the concentrations of monomer, and growing radicals. The concentration of growing radicals is related to the attention of dormant species, activators, and deactivators. Equation 1 shows fast initiation and no chain termination under the ideal case. The dispersity of molecular weight (MW) (Mw/Mn) of polymers influenced by dormant groups' concentration ( $\mathrm{P}_{\mathrm{n}} \mathrm{X}$ and $\left.\mathrm{X}-\mathrm{CuII}\right)$, the rate constants of propagation $\left(\mathrm{k}_{\mathrm{p}}\right)$, and deactivation $\left(\mathrm{k}_{\text {deact }}\right)$, and monomer conversion $(\mathrm{p})$.

$$
\mathrm{R}_{\mathrm{p}}=\mathrm{k}_{\mathrm{p}}[\mathrm{M}]\left[\mathrm{P}_{n}^{*}\right]=k_{p} \mathrm{~K}_{\mathrm{ATPR}}\left(\frac{\left[\mathrm{P}_{\mathrm{n}} \mathrm{X}\right]\left[\frac{\mathrm{Cu}}{\mathrm{L}}\right][\mathrm{M}]}{\left[\mathrm{X}-\frac{\mathrm{Cu}^{\mathrm{II}}}{\mathrm{L}}\right]}\right)
$$

\subsubsection{RAFT polymerization}

The reversible addition-fragmentation chain transfer (RAFT) process has been a powerful and popular polymerization technique in the modern field of complex polymeric synthesis processes $[11,12]$. RAFT belongs to reversible deactivation radical polymerization (RDRP), also called living radical polymerization, and it has the ability to predictable molecular weight and low molar mass dispersity. The RAFT mechanism (Figure 3 and Figure 4) is shown below. In step I, the radical source is added to the RAFT. It will enter to equilibrium between active and dormant species in later steps III and V. In the RAFT mechanism, which formed by the chain transfers steps, the reactions degenerate due to the involvement of eversible transfer of the functional chain end-group. For example, the thiocarbonylthio group, $\mathrm{Z}-\mathrm{C}(=\mathrm{S}) \mathrm{S}-\mathrm{R}$. In the result part, all chains will result in a similar degree of polymerization (DP). And the whole process is based on the insert of monomers between the R- and $\mathrm{Z}-\mathrm{C}(=\mathrm{S}) \mathrm{S}$ groups of a RAFT agent and formed $\alpha$ and $\omega$ end-group at the resulting polymeric chains. However, using the $\omega$ - 
functionalization is not the best approach in some applications due to the instability of the $\mathrm{C}-\mathrm{S}$ bond in the RAFT end-group.

The critical element in the RAFT mechanism is based on the correspondence between the number of chains that undergo bimolecular termination and the number of initial radicals in the whole system. Indeed, unlike other systems, the bimolecular termination event will not cause the loss of the $\omega$-end thiocarbonylthio end-group, so the total amount of thiocarbonylthio end-group is constant in the whole process. As a result, the number of dead chains can be predicted and controlled by the number of radicals introduced in the system.

$$
\text { II. }
$$

\section{Stimuli-responsive polymeric micelles}

Nowadays, to enhance the efficient drug release rate in the tumor cells, the targeting drug release mechanism is developed in polymeric micelle system by controlling the methods of drug release when reach the target cells [13]. The stimuli-responsive of polymeric micelle has been used as an innovative technology for possession of drug release. The stimuli systems can be divided into two main parts: internal stimuli and external stimuli. For internal stimuli, it contains temperature, $\mathrm{pH}$-value, enzymes, and so on. In this method, compound Thermo or $\mathrm{pH}$-sensitive compound releases the inside drugs after reaching the targeting tumors. The external stimuli methods include light, ultrasound, and both types of stimuli-responsive methods show a superior ability on cancer therapy on accuracy on location and time of drug release.

\section{1. $\mathrm{pH}$-sensitive polymeric micelles}

During the cancer therapy process, tumor microenvironment is one of the most important factors that can influence treatment. Tumor microenvironment (TME) demonstrates a tumor composition, and it has been focused as a highly effective technique to enhance human immunotherapy [14]. PH value has been considered an ideal trigger for anticancer drug release, based on the different $\mathrm{pH}$ values between primary tumors and normal tissue [15]. In a human vivo system, many intracellular components can maintain their $\mathrm{pH}$ value in normal conditions, such as cytoplasm, endosomes, lysosomes, and mitochondria. When the tumor overgrown, the expanding population of tumor cells caused the lack of oxygen. This reaction results in hypoxia, lactic acid production, and hydrolysis of ATP in the human Vivo system. Therefore, the acidic microenvironment can be direct to a $\mathrm{pH}$-sensitive drug delivery system developed. The $\mathrm{pH}$ value in tumor cells is $\sim 6.5$, and the value of normal cells is $\sim 7.4$, and $\mathrm{pH}$-value can decline further in the organelle such as endosomes and lysosomes [16]. In this way, planning the $\mathrm{pH}$-delicate nanocarrier for focusing on drug conveyance to tumors that the nanocarrier is steady at physiological $\mathrm{pH}$; however it should be distorted to work with the arrival of the medication under gentle acidic conditions at target tumors, may bring about essentially upgraded remedial adequacy and insignificant results [17].

\subsubsection{Unimolecular polymeric micelles (UPM)}

In this manuscript, the drug delivery systems of a $\mathrm{pH}$ sensitive polymeric micelle are triggered by the protonation of carboxylic acid group. The $\mathrm{pH}$ value of polymeric micelle must be stabled at 7.4 to reduce the probability of the disassembly and destabilization in vivo. To prepare $\mathrm{pH}$-sensitive polymeric micelle, positivecharged drugs are combined with negative-charged block copolymer to form a core. In the human vivo system with neutral $\mathrm{pH}$ value, the carboxylic acid units are shown as negative charge, allowing cooperative electrostatic interactions with the encapsulated compounds [18]. Once in a mildly acidic environment, protonation of the carboxylic acid gatherings brings about a net reduction in electrostatic interactions resulting in separation of the medication-containing centre. Another way to produce $\mathrm{pH}$-sensitive PMs is to combine a drug in the uncharged hydrophobic centre introducing an ionized polyanion shell. For this situation, protonation of the body prompted an annoyance of the centre shell structure and eventually influenced the intracellular conveyance of the medication. 
There are mainly two $\mathrm{pH}$-stimuli polymeric micelle types that are commonly used: unimolecular and multimolecular [18].

Amphiphilic star-shaped polymers were a novel method to the $\mathrm{pH}$-sensitive of polymeric micelle by atom transfer radical polymerization. The new polymers show a traditional core-shell structure of PM, and have dilution stability as unimolecular polymeric micelles (UPM). UPM can be extracted from both dendrimers and star polymers (Figure 5A). A four-armed multifunctional initiator was used for polymerization, as the Star polymers can be produced in fewer steps than other technology. Atom transfer radical polymerization (ATRP) was used to synthesize UPM in the previous work (Figure 5B). A novel water-soluble UPM with an ionizable core was developed as potential carriers for the oral delivery of drugs. The compound was called Star-P(EMA-cotBMA)-b-P(PEGMA). To investigate the function of UPM, the $\mathrm{pH}$-value of different concentrations and percent of invitro drug release rate were tested. Titration was used to evaluate MAA content with hydrolyzed UPM and $\mathrm{NaOH} 0.05 \mathrm{~N}$. The value of $\alpha$ shown the degree of
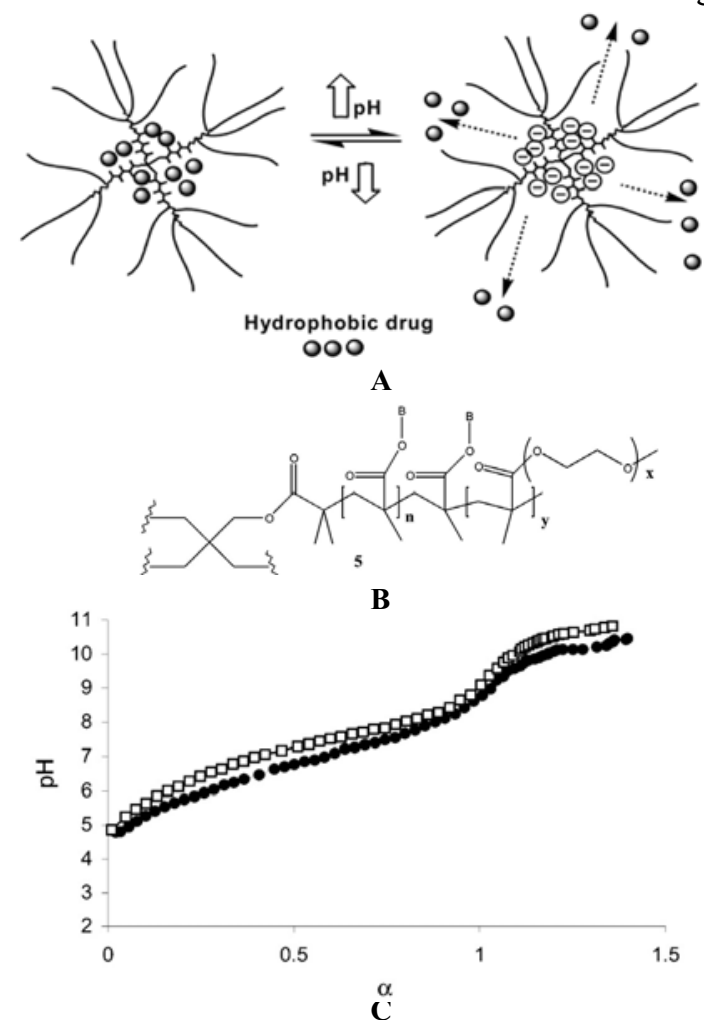

Figure 5. (A) The structure of $\mathrm{pH}$-sensitive unimolecular polymeric micelles (UPM) release hydrophobic drug. (B) The chemical structure of pH-sensitive UPM. (C) The comparison of pH-value and UPM concentration of S_E18M17P442 (closed circles) and S_E24T23P229 (open squares). $\alpha$ was the ionization, and the polymer solution was titrated with $\mathrm{NaOH}$ solution. (D) (a) In $\mathrm{pH} 1.2$ environment, vitro release of free (closed triangles) and S_E24M23P229-entrapped (5\% w/w) (open triangles). (b)In pH 1.2, 7 (open squares) and 11 (closed circles) environment, S_E24M23P229-entrapped progesterone $(5 \% \mathrm{w} / \mathrm{w})$ released.

\subsubsection{Unimolecular polymeric micelles (UPM)}

$\mathrm{pH}$-sensitive polymeric micelle also can be used as the $\mathrm{pH}$ probe for detecting acidic biological environment. $\mathrm{pH}$ imaging for tumor therapy was due to the unique acidic environment. To monitor tumor $\mathrm{pH}$-value in the human vivo system, MR imaging/spectroscopy (MRI/MRS) was used as clinical applicability [19]. To reach a better $\mathrm{pH}-$ ionization, where $\alpha=\mathrm{Cb} / \mathrm{CMAA}$ and $\mathrm{Cb}$ is the concentration of $\mathrm{NaOH}, \mathrm{CMAA}$ is the concentration of MAA(methacrylic acid) in solution. The result shows that the titration curve reaches the equivalent point when $\mathrm{pH}=9$. Indeed, it also shows that UPM can be protonated in the stomach ( $\mathrm{pH} 1$ to 2), Ileum ( $\mathrm{pH} 7$ ). As the drug release rate depends on drug diffusion, the stability of carrier material, and polymer degradation, the introduction of $\mathrm{pH}$-sensitive acidic functions were supposed to influence the polarity of $\mathrm{pH}$ response. In Figure 5D (a), the graph shows the percentage of drug release rate is between free drugs and packed drugs. After $60 \mathrm{~min}$, more than $80 \%$ of non-packing drug release, and the diffusion rate reach $100 \%$ after $2 \mathrm{~h}$. Compared with the rate of drug release from UPM, it hardly reaches $20 \%$ after $60 \mathrm{~min}$, and the maximum rate is $45 \%$ in $8 \mathrm{~h}$. In figure 5D (b), the graph showed the $\mathrm{pH}$-sensitive drug from UPM in $\mathrm{pH} 7$ or 11 conditions. Based on the graph, the change of drug release amount from the micelles at higher $\mathrm{pH}$ was shown.
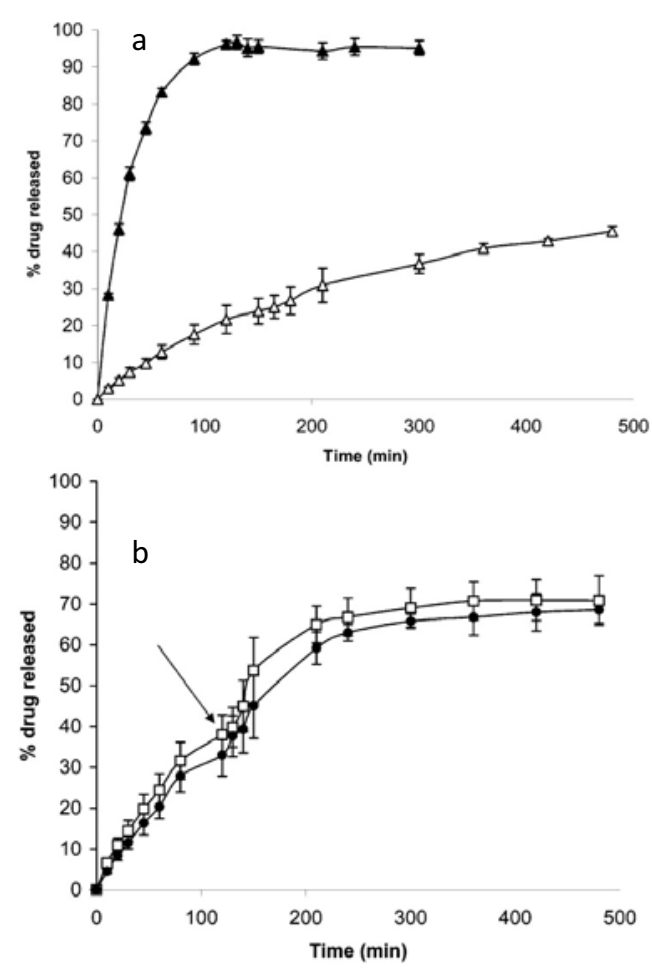

D polyethylene glycol (PEG)-b-poly (l-histidine) (PHis) and PEG-b-poly (1-lactic acid) (PLLA) (Figure 6A).

To get a better result of tumor testing using MRs, the $\mathrm{pKa}$ value of the $\mathrm{pH}$ probe should range between weak acidic and neutral (pH 6.0-7.4). The PHis material can be changed from water-soluble to hydrophobic aggregates with increasing $\mathrm{pH}$-value. As the $\mathrm{pH}$-value and pass the sensitive system, a mixed micelle $\mathrm{pH}$ probe composed of 
$\mathrm{pH} 7.0$ condition, the normalized integration percentage of the $\mathrm{H} 2$ peak decrease sharply, as $\mathrm{H} 2$ represented the chemical shift imidazole ring. With the molecular weight increase, the aqueous solubility of PHis is decreased (Figure 6B). The function of micelle probes was tested by using $1 \mathrm{H}-\mathrm{MRS}$ to compare the $\mathrm{pH}$-value and chemical shift of the H2 peaks in the graph. The $\mathrm{pH} 7.4$ and 6.0 conditions were used to clarify the MRS data of the mixed micelles (Figure 6C). At $\mathrm{pH}=6.0, \mathrm{H} 2$ and $\mathrm{H} 5$ peaks of the mixed micelle showed a visible rise compared with the $\mathrm{pH}$ 7.0 condition. The diverse micelle probe peaks coincided with the spectrum of blood itself. The results indicated the mixed micelle can provide $\mathrm{pH}$-monitoring power in tumor $\mathrm{pH}$-value range.

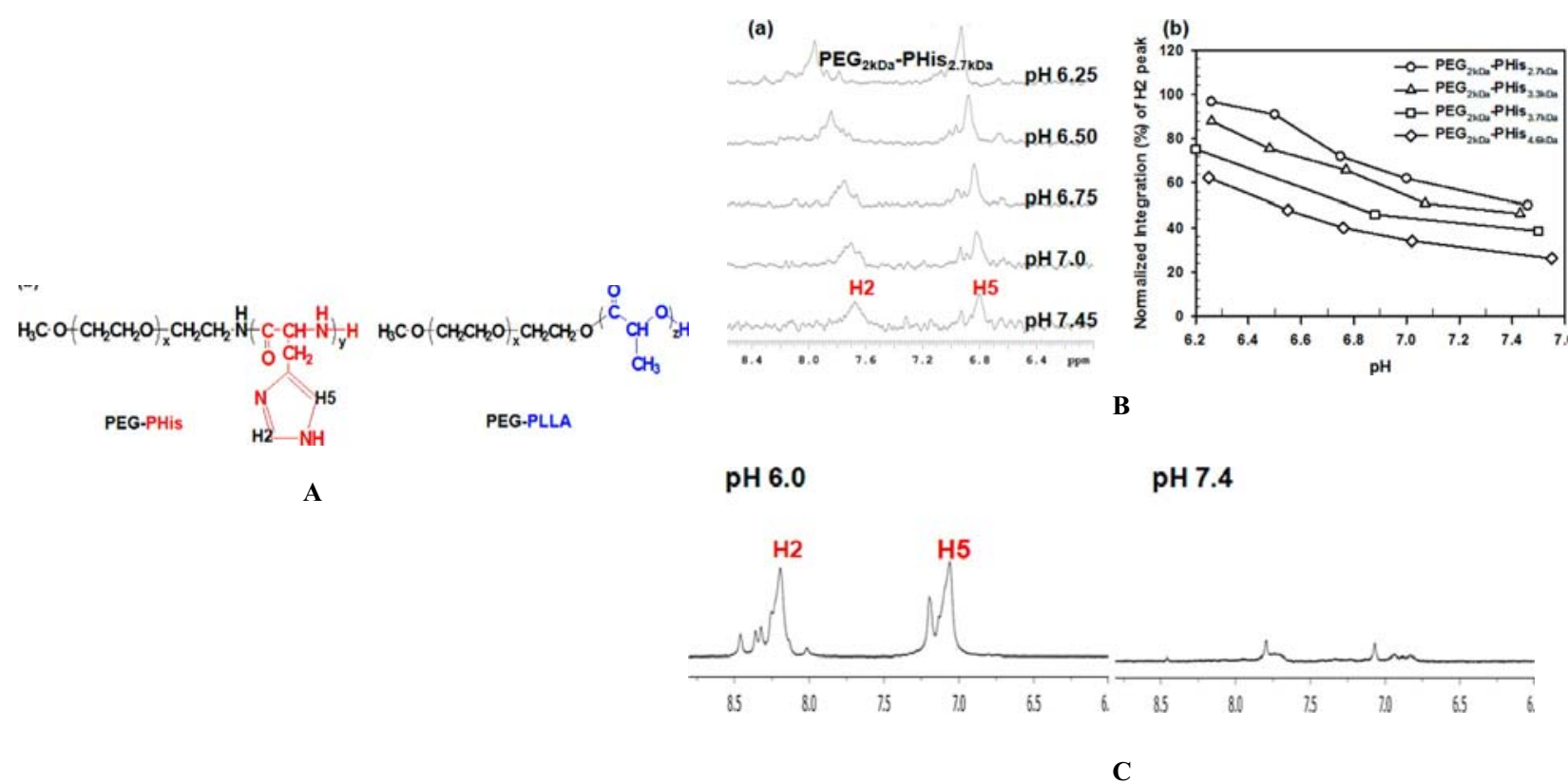

Figure 6. (A) The chemical structure of polyethylene glycol (PEG)-b-poly (1-histidine) (PHis) and PEG-b-poly(l-lactic acid) (PLLA). (B) (A) $\mathrm{H} 2$ and $\mathrm{H} 5 \mathrm{HNMR}$ peaks in PEG2kDa-PHis2.7kDa at different $\mathrm{pH}$ conditions. (B) $\mathrm{pH}$-sensitive $\mathrm{H} 2$ and $\mathrm{H} 5$ peaks of PEG2kDa-PHis in different MWs of PHis. (C) $1 \mathrm{H}$ NMR spectra of $\mathrm{H} 2$ and $\mathrm{H} 5$ peaks in mixed micelles of PEG2kDa-PHis3.3kDa (90 wt \%) having PEG2kDa-PLLA3kDa (10 wt \%) in different $\mathrm{pH}$ condition (6.0 and 7.4).

\subsection{Photo-sensitive polymeric micelles}

For the photosensitive method to control the polymeric micelle remotely and accurately, which can focus into specific areas quickly. Based on modern technology, the use of light control factor for polymeric micelle was largely unexploited [20].

\subsubsection{Poly(tert-butyl acrylate-co-acrylic acid) (tBA-AA)}

Synthesis of an amphiphilic diblock polymer micelle was developed to stimulate by UV light and be reformed by later light exposure. In the synthesis of polymer micelles, it can be divided into two parts. For the hydrophobic block, its principal structure is a side-chain liquid crystalline polymethacrylate with azobenzene mesogens (PAzo). And the hydrophilic block is a random copolymer of poly(tert-butyl acrylate-co-acrylic acid) (tBA-AA) [21]. The hydrophobic, which is the core part, contains a photolabile chromophore as a pendent group to result in the polymeric micelle break under UV light. The bondbreaking reaction occurred between the chromophore and polymer under UV light and resulted in transformation of a hydrophobic block into a hydrophilic block.

To detect the bond-breaking and dissociation of polymeric micelle in UV light condition, scanning (SEM) and transmission electron microscope (TEM) technology were used to observe the reaction in the solution. The polymeric micelles were formed in the solution in nonUV light conditions, and the compared group was placed under UV light. In the result, no micelles remained after exposure to UV light condition, which indicated the polymeric micelle probe can monitor the dissociation of micelle (Figure 7A). Meanwhile, as the intensity of the irradiation increased, the transmittance increased. As the dissociated of micelles under UV light, the solution turbidity rate decline contributes to the rise of transmittance rate (Figure $7 \mathrm{~B}$ ). The result showed the detachment of dye pendant groups responded to photo solvolysis. 
a)

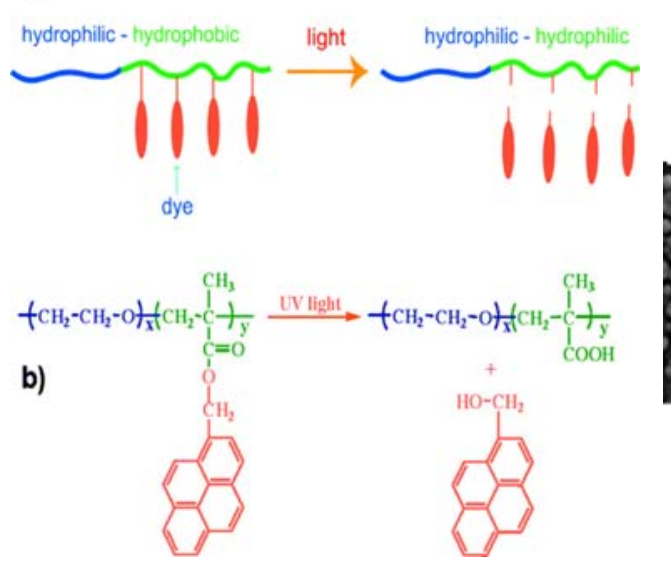

A (a)
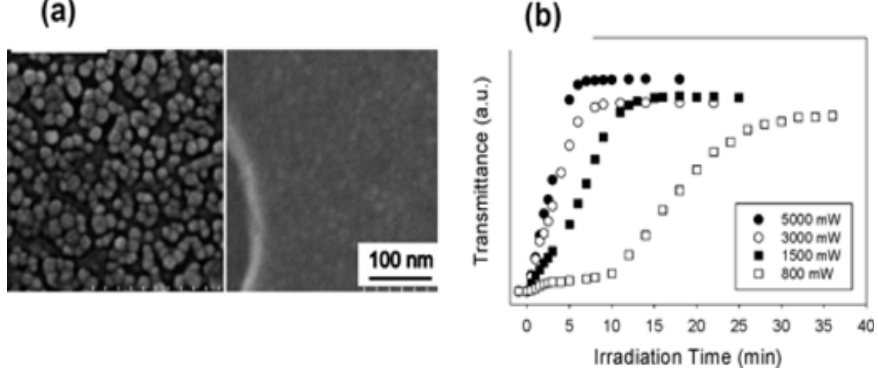

B

Figure 7. (A) (a) The flow chart of the change of hydrophobic to hydrophilic due to detachment of dye pendant groups. (b) Chemical structure of the amphiphilic diblock copolymer and reaction under UV light. (B) (a) The scanning image of PEO-b-PPy (left) and the dissociation of micelles under UV light in the micellar solution(right). (b) The comparison of transmittance rate and time under different irradiation intensities.

\subsubsection{UCNPS}

Many photosensitive required a high-energy UV light during the reaction; however, the high intensity may induce negative effects on the human body. So longerwavelength near-infrared (NIR) light, which has less penetration into cells, was a modern popular field. In the photoreaction, NaYF4:TmYb upconverting nanoparticles (UCNPs) was encapsulated in poly(ethylene oxide)block-poly(4,5-dimethoxy-2-nitrobenzyl methacrylate) micelle under 980nm light [22]. By encapsulating $\mathrm{NaYF} 4: \mathrm{TmYb}$ upconverting nanoparticles (UCNPs) inside micelles of poly(ethylene oxide)-block-poly(4,5dimethoxy-2-nitrobenzyl methacrylate) and exposing the micellar solution under $980 \mathrm{~nm}$ light, photons are emitted by the UCNPs. During the mechanism, photons are absorbed by o-nitrobenzyl groups on the micelle coreforming block, activating the photocleavage reaction and leading to the dissociation of BCP micelles core and release of co-loaded hydrophobic species (Figure 8A).

To determine NIR light whether can induce the release of hydrophobic species co-loaded with the UCNPs inside the micelles, micelles contained UCNPs and Nile Red (NR) were used to observe the result. The absorbance spectrum recorded the UCNP-loaded micellar solution before and after NIR light condition without NR add. No changes in absorbance without irradiation in $24 \mathrm{~h}$, so the result convinced the photocleaved when micelle break under NIR light (Figure 8B(a)). For the micelles loaded contain UCNPs and NR, the normalized fluorescence intensity of NR was measured under NIR light at $640 \mathrm{~nm}$, and the result showed a hydrophobic dye is much faster under NIR light of the micellar solution (Figure 8B(b)).

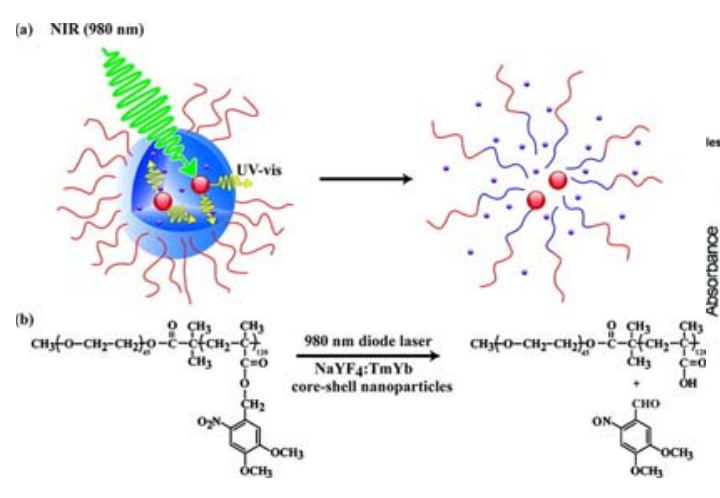

$\mathbf{A}$
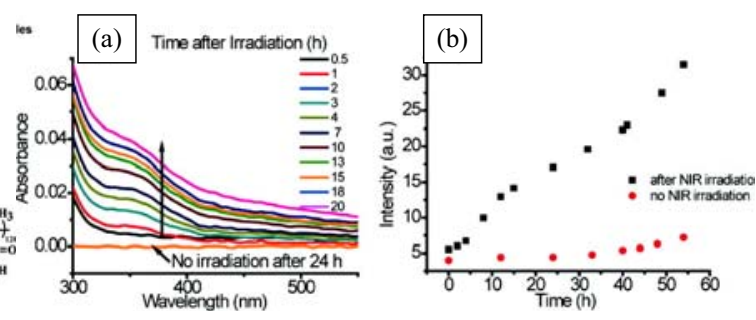

B

Figure 8. (A) (a) The dissociation of BCP micelles in UCNPs under NIR light. (b) The photoreaction trigged by NIR light with BCP of PEO-b-PNBMA and UCNPs of NaYF4:TmYb. (B) (a) Absorption spectrum vs. time of dissociation of UCNP-loaded micelles under NIR light exposure (5W, 4h). (b) Intensity rate in the normalized fluorescence at $640 \mathrm{~nm}$ vs. time for two micelle-loaded solutions contain NR and UCNPs. Black dot was subjected under NIR light condition, and the red dot was not. 


\subsubsection{Gemcitabine (GEM)}

Light-cross-linked small-molecule micelles with enediyne units are designed to increase the efficiency of the drug delivery rate. Gemcitabine (GEM) was used as hydrophilic drug, and it can tethered with a maleimidebased enediyne (EDY), which can be used hydrophobic tail in the preparation of amphiphilic EDY-GEM under Bergman cyclization (Figure 9A). The process is an intramolecular cyclization of EDY under photo-triggering conditions and formed diradical type intermediates [23]. Confocal laser scanning microscopy (CLSM) technology had the ability to show the drug-loaded micelles and lightcross-linked micelles. Human cell lung cancer (A549) cells were used to react with light-cross-linked micelles $(50 \mu \mathrm{M})$ in $6 \mathrm{~h}$ and shown fluorescence image (Figure 9B). In green and blue images $(525 \mathrm{~nm}$ and $450 \mathrm{~nm})$, the cellular uptake was established, which indicated the successful delivery of the drug (GEM) to the cancer cells and nucleus.

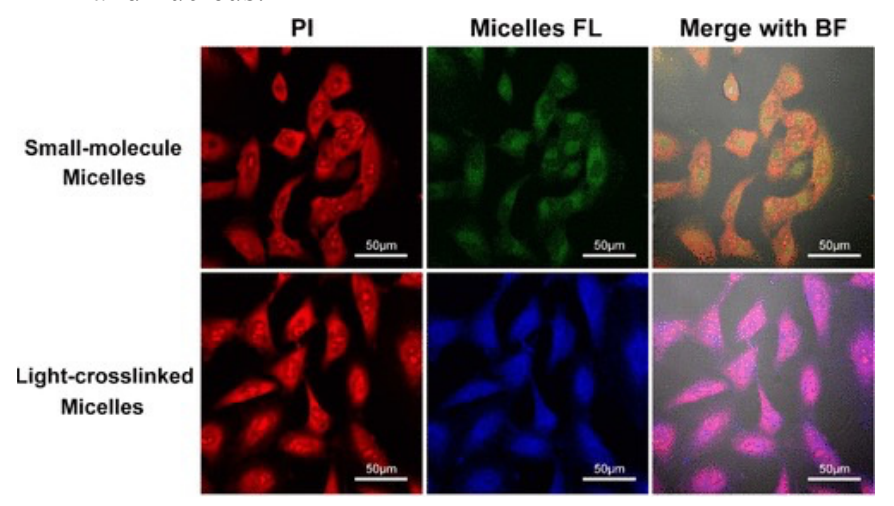

A

Figure 9. (A) light-cross-linked small-molecule micelles chemical structure (6). (B) CLSM image of A549 with light-cross-linked micelles for $6 \mathrm{~h}$. The cell dyed with propidium iodide (red), the micelles fluorescence in cells (green/blue), and overlapped two images into 3 th column image. The concentration of [EDY-GEM] was $50 \mu \mathrm{M}$.

\subsection{Temperature-sensitive polymeric micelles}

Temperature plays a critical role in nearly everything in chemical reaction and regulation, for example, reaction requirement, cell division, and gene expression [24]. Compared with other external stimuli, such as $\mathrm{pH}$-valued, light and so on, temperature control is one of the simplest drug release methods. The basic structure of thermosensitive polymeric micelle was formed by hydrophobic block and thermo-responsive hydrophilic block.

\subsubsection{Poly(N-isopropylacrylamide) (PIPAAm)}

Poly(N-isopropylacrylamide) (PIPAAm) is hydrophilic and known as a characteristic reversible temperatureresponsive phase transition when it reaches the lower critical solution temperature (LCST) in aqueous [25]. By adding hydrophilic comonomers, such as N,Ndimethylacrylamide, the value of LCST of PIPAAm could be easily controlled to a near body temperature (Figure 10A).

To ensure the efficiently influenced efforts between time and temperature in the intracellular uptake, the micelles were incubated at two temperatures, $37{ }^{\circ} \mathrm{C}$ (below the LCST) and $42{ }^{\circ} \mathrm{C}$ (above the LCST) (Figure 10B). The result of fluorescence intensity showed the amount of intracellular increased sharply only when temperature above micelle LCST. This result is caused by the enhancment of the interaction between micelle and cells due to the thermo-sensitive phase of the micelle coronas.
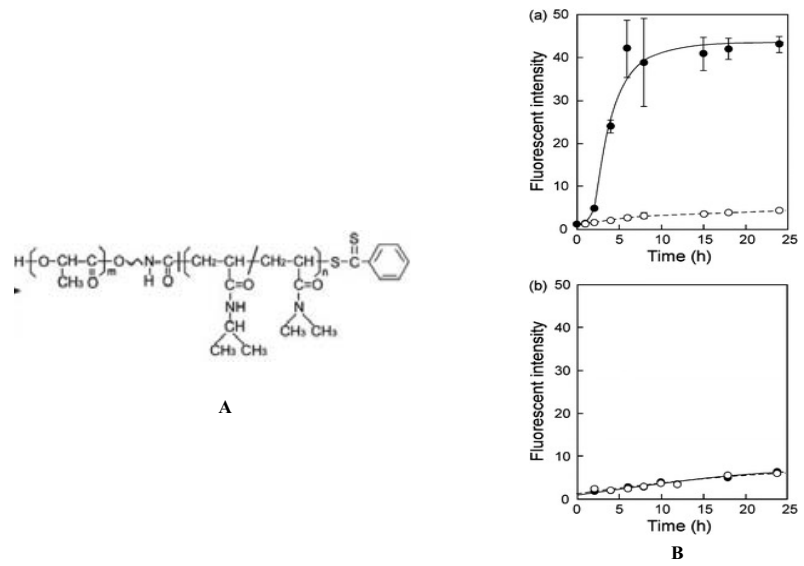

Figure 10. (A) The chemical structure of polymeric micelle from thermo-sensitive polymer. (B) Comparison of time and fluorescent intensity of (a) OG-labelled thermo-responsive micelles and (b) the OG-labelled P(IPAAm-co-DMAAm) in different temperatures $\left(37^{\circ} \mathrm{C}\right.$, open circle) and above $\left(42^{\circ} \mathrm{C}\right.$, closed circle).

\subsubsection{P(IPAAm-DMAAm)-b-PLA)}

One kind of mechanism of thermo-responsive polymeric micelles is motivated by poly ( $\mathrm{N}$-isopropylacrylamide-coN,N-dimethylacrylamide)-b-poly(d,l-lactide) (P(IPAAmDMAAm)-b-PLA (Figure 11A), its LCST can be modulated for desirable temperatures, by the introduction of the hydrophilic or hydrophobic monomers into the PIPAAm leading chains [26]. Based on the coronaforming, the temperature-sensitive polymeric micelle can 
reversibly change structure and water solubility across the change on LCST.

The property of temperature-sensitive can influence the drug release rate of polymeric micelle. By controlling the variable of temperature in the range of $37-45^{\circ} \mathrm{C}$, the viable change in the fluorescent intensity occurs at $39.4{ }^{\circ} \mathrm{C}$ (Figure 11B). When the heat is provided above the LCST, the fluorescent intensity increases when the temperature increases to in the range of $39-43^{\circ} \mathrm{C}$. Also, a significant decrease in the percentage of cell viability was observed around $45^{\circ} \mathrm{C}$, and this result may cause by the extremely higher than limited temperature, so critical
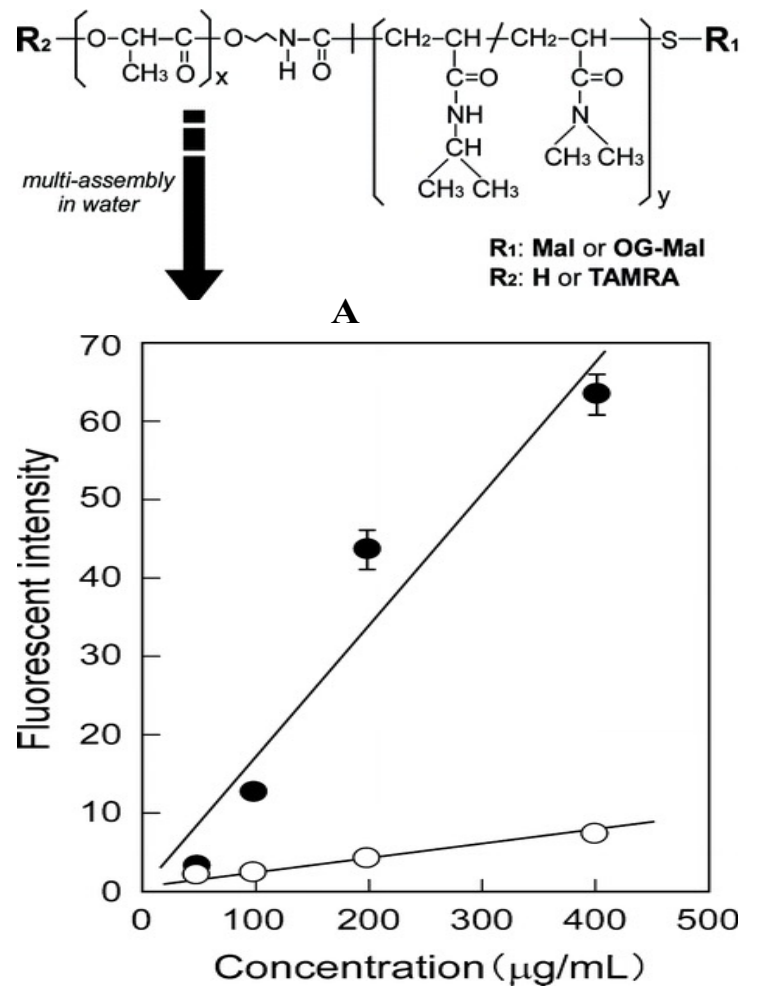

B destruction of cellular functions of cellular proteins occurs. The sharply decrease cellular viability when the temperature approaches $45^{\circ} \mathrm{C}$ also illustrated this point.

The repeated cycle of the intracochlear uptake of P(IPAAm-DMAAm)-b-PLA micelles in the temperature range of 37 to $42{ }^{\circ} \mathrm{C}$ (Figure 11C). The intracellular micelle uptake, which was indicated by the fluorescent intensity, was significantly increased by heating above the LCST. Moreover, when the LCST reaches $37{ }^{\circ} \mathrm{C}$, the micellar internalization is limited due to P(IPAAmDMAAm) reversibility, and these results achieved the temperature control intracellular micelle uptake.

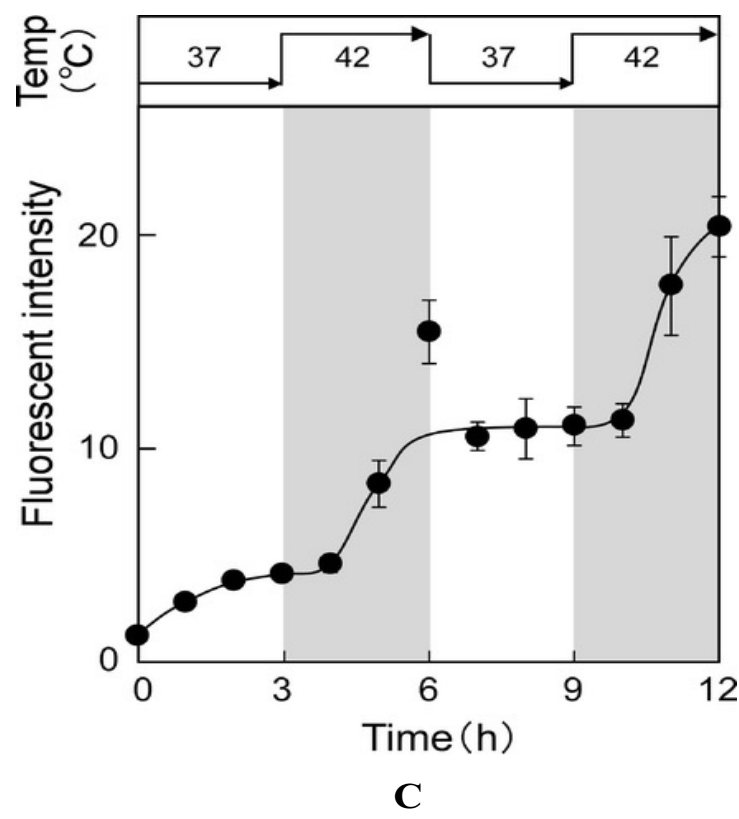

Figure 11. (A) Chemical structure of thermo-responsive block copolymers of P(IPAAm-DMAAm)-b-PLA. (B) The temperature effect the P(IPAAm-DMAAm)-b-PLA micelles (closed circles) and cellular viability (open circles). Cells were incubated with DMEM/FBS, including $200 \mu \mathrm{g} / \mathrm{mL}$ of OG-labelled P(IPAAm-DMAAm)-b-PLA micelles for $6 \mathrm{~h}$ at different temperatures. (C) The time effect the fluorescent intensity of P(IPAAm-DMAAm)-b-PLA micelles (closed circle) in the range of temperature between 37 and $42{ }^{\circ} \mathrm{C}$. The micelles concentration is $200 \mu \mathrm{g} / \mathrm{mL}$ in DMEM/FBS.

\subsection{Reduction control}

Reduction-sensitive polymeric micelle is one of the modern class of biomedical material which can be applied for drug delivery. It can remain excellently stable in human circulation and in extracellular fluids, and in intracellular compartments, for example, cytoplasm and the cell nucleus, micelle can be easily degraded [27]. The common structure of reduction-sensitive polymers usually contains disulfide linkage(s) in the main chain, side chain, or cross-linker. In standard conditions, the disulfide bonds are stable in the circulation and in the extracellular milieu. However, under thiol-disulfide exchange reactions, the bond can be cleavaged from minutes to hours [8]. For example, compared with other polymers, aliphatic polyesters and polycarbonates take a long range of time to cleavage.

\subsubsection{HECS-SS-OA}

An O,N-hydroxyethyl chitosan-octylamine (HECS-ssOA) was introduced as a conjugate to build disulfide linkages between the hydrophobic alkyl chains and hydrophilic chitosan backbone. And O,N-hydroxyethyl chitosan-octylamine conjugate (HECS-ss-OA) involving bioreducible 3,3'-dithiodipropionic acid (DPA), resulted in the formation of a redox-sensitive micellar system [28]. This system was used to trigger intracellular delivery of hydrophobic paclitaxel (PTX). In the experiment, a control group insensitive structurally analogous HECSOA conjugates was synthesized, which is structurally 
similar to HECS-ss-OA, but lacked the redox-sensitive disulfide bond (Figure 12A and 12B). The disassembling process of the reduction-sensitive polymeric micelle was triggered by GHS, which is a natural reducing agent for disulfide bonds, based on the size change of the HECSss-OA and HECS-OA micelles. GHS is possibly connected with cancer, as the concertation of the GHS in tumor was found extremely higher than in normal cells. The mean of the size of the HECS-ss-OA micelles, which is the peak of the curve, increased when $10 \mathrm{mM}$ or $20 \mathrm{mM}$ GSH was added. And the rate of increase in mean particle size was controlled by the concentration of GHS. The result indicated the redox-sensitive micelles have the ability to remain stable in normal conditions, but in a high reducing agent concentration, micelles will be disassembled, which indicated the suitability of redoxsensitive micelles for specific redox-mediated intracellular delivery of anticancer drugs. The tendency of the curve indicated cumulative release percentage curves of PTX from the micelles at variable GSH concentrations. The PTX release from the HECS-ss-OA micelles was slow when the GSH level $(10 \mu \mathrm{M})$ is low, and only $15.8 \%$ of PTX was released after 6 hours incubation. After 24h, $21.3 \%$ was released (Figure 12C). However, under high concentrations of GSH $(20 \mu \mathrm{M}$ simulating the tumor GSH concentration, the drug release rate from the redoxsensitive micelles increased sharply. For example, under $20 \mathrm{mM}$ GSH condition, approximately $71.3 \%$ cumulative release was observed after 6 hours, and $95.4 \%$ release rate was observed after 1 day. The reduction-sensitive polymeric micelle can be rapidly released in the intracellular tumor environment, due to the explosion to high concentrations of GSH.

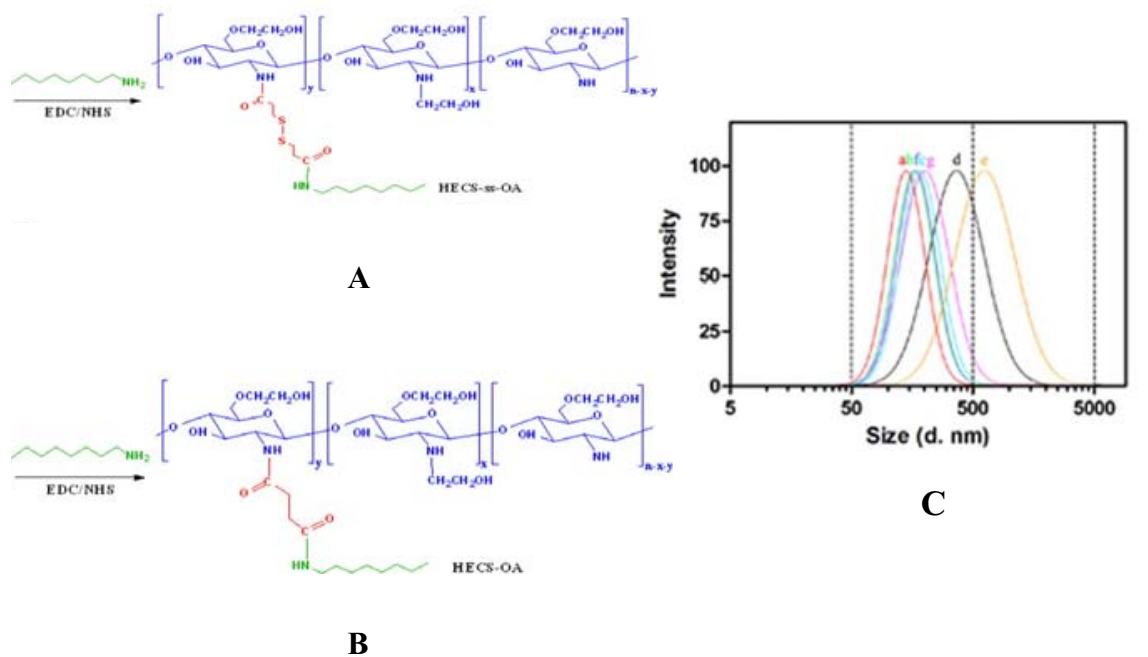

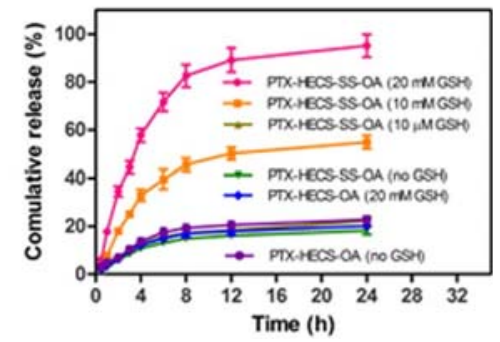

D

Figure 12. (A) The chemical structure of redox-sensitive HECS-ss-OA conjugate. (B) Non-redox-sensitive HECS-OA conjugate. (C) The comparison of size HECS-OA micelles and HECS-ss-OA micelles and intensity at different concentrations of GSH determined by DLS for 36h. (a) HECS-ss-OA micelles before GSH addition. (b) HECS-ss-OA micelles in the absence of GSH. (c) HECS-ss-OA micel fff le s were incubated alongside $10 \mu \mathrm{M} \mathrm{GSH}$. (d) HECS-ss-OA micelles were incubated alongside $10 \mathrm{mM}$ GSH. (e) HECSss-OA micelles were incubated alongside $20 \mathrm{mM}$ GSH. (f) HECS-OA micelles before adding GSH. (g) HECS-OA micelles incubated alongside $20 \mathrm{mM}$ GSH. (D) The plot of time (h) vs. the $\mathrm{p}$ ercentage of cumulative release of the release of HECS-OA micelles and HECS-ss-OA micelles trigged by GSH. The standard deviation represents in the error bar $(n=3)$.

\subsection{2 folic acid-decorated amphiphilic polymer-CPT}

In the current study, folic acid (FA)-decorated amphiphilic polymer-CPT conjugates with disulfide linkages (FSC) were used to design novel reductionsensitive polymeric micelles (Figure 13A). The active CPT was released by the linker between CPT and amphiphilic poly(ethylene glycol)-b-poly(e-caprolactone) (PECL) copolymers to respond to GSH [29]. FA has been used widely, due to its property of accurate target to tumor cells, and the ability to avoid normal nonspecific attacks on normal tissues. The polymeric micelles were produced by FSC and FC conjugates. Moreover, FSC-16 micelles were synthesized from hexadecanol, and FSC (or FC) conjugates were dissolved in THF. To examine the responsiveness, the CPT release from FSC and FSC-16 micelles were investigated in the presence or absence of GSH and compared with those of FC and FC-16 micelles.
GSH concentrations of $2 \mu \mathrm{M}$ and 10 and $40 \mathrm{mM}$ were used to represent the reductive microenvironment of extracellular space and cytoplasm of normal and tumour cells, respectively.

To determine the responsiveness of the reductionsensitive polymeric micelles in tumor cells, the CPT release from FSC and FSC-16 micelles was investigated under contains GHS or no GHS condition. The concentrations of GHS included $2 \mu \mathrm{M}$ and 10 and $40 \mathrm{mM}$ were used to represent the environment of extracellular space and cytoplasm of normal and tumor cells. The rate of CPT release from FSC micelles is influenced by the concentration of GHS positively (Figure 13B). Meanwhile, in $10 \mathrm{mM}$ GHS condition, $38 \%$ of CPT release was detected for FSC micelles after incubation $100 \mathrm{~h}$, shown the reductive sensitivity of CPT release. Advantages in premature release during circulation can influence cellular uptake efficiency in cancer cells and promote the intracellular release by designing amphiphilic 
copolymers containing reduction-sensitive linkages between CPT and polymers. And FA is used as a targeting section.

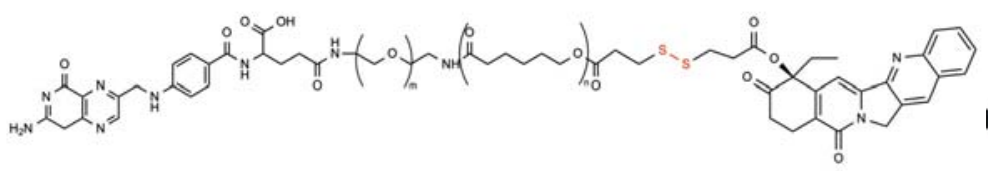

FA-PECL-SS-CPT

A

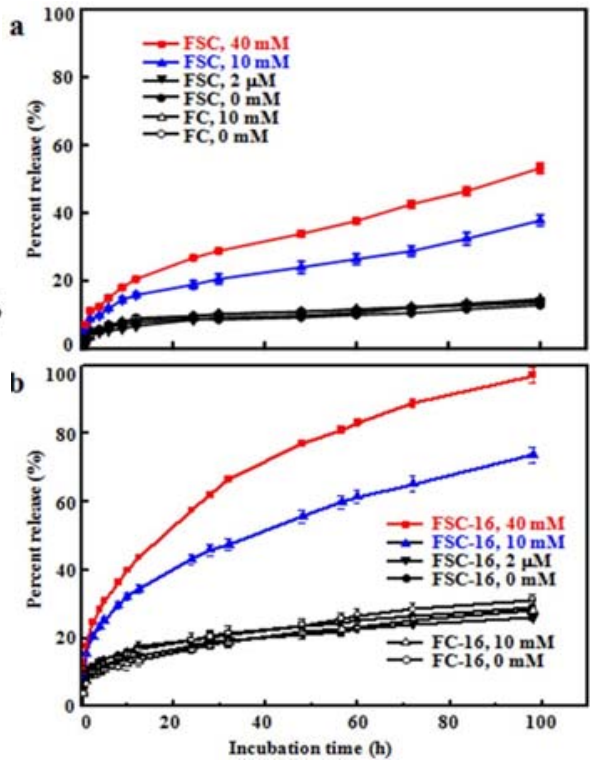

B

Figure 13. (A) The chemical structure of FA-decorated polymer-drug conjugates containing disulfide linkages (red line) between CPT and amphiphilic PECL copolymers. (B) The comparison of incubation time and percentage release of CPT from (a) FSC and (b) FSC-16 micelles incubation at $37^{\circ} \mathrm{C}$ in standard PBS and PBS with $2 \mu \mathrm{M}$ and 10 and $40 \mathrm{mM} \mathrm{GSH}$, compared with FC and FC-16 micelles without disulfide linkage.

\section{Modern technology}

Recent technological advancements have fostered the use of polymeric micelles as drug delivery systems, particularly for lipophilic drugs with several benefits such as small size(10-100nm), prolonged time circulation, enhanced accumulation of drugs at tumor sites, and shellcore structure. Due to polymeric micelles being relatively more stable compared to surfactant micelles, it is preferred for its ability to solubilize hydrophobic molecules in their main core. They are also being used to transport antisense, plasmid DNA, oligonucleotides, and deliver diagnosis agents to particular organs in the body.

Polymeric micelles have emerged as potential carriers of poorly water-soluble drugs, and they provide attractive traits. For that reason, new technologies have led to the development of several methods for preparing polymeric micelles, such as the dialysis method, solid dispersion, oil in water emulsion, and microphase separation method. The dialysis methods involve adding water to the polymer solution and drugs in water-miscible organic solvents. The solid dispersion methods are conducted by dissolving the polymer and the drug in an organic solvent to obtain a solid polymer matrix after the evaporation of the solvent under reduced pressure. The oil in water emulsion solvent evaporation approach involved dissolving drugs and polymers in water-immiscible organic solvents and slowly adding distilled water under vigorous stirring to rearrange the polymer to create micelles. Ultimately, the microphase separation method is conducted by dissolving the polymer and the drug in an organic solvent and adding the solvent in water under magnetic stirring to spontaneously for polymeric micelles.

\section{Conclusion}

Polymeric micelles have been subject to various scientific studies over the last decade. They have been studies as versatile and efficient drug delivery systems. For that reason, new designs have been developed to create polymeric micelles to increase drug loading capacities. Various strategies have been formulated to enhance the interaction of polymeric micelles with carriers to maximize loading capacities, such as hydrotropic polymers. However, there is a need for more systematic studies on polymeric micelle structures and their activity relationships to enhance the understanding of the mechanisms of polymeric micelles and their interactions with carriers and the effect of their structure on the loading capacity of carriers.

\section{References}

1. T. Millera, S. Breyer, G.Colena, W. Mier, U. Haberkorn, S. Geissler, S.Voss, M. Weigandt, A. Goepferich, I.J.Pharm 445, 118 (2013) 
2. H. Cho, T. Lai, K. Tomoda, G. S. Kwon, AAPS PharmSciTech 16, 13 (2015)

3. C. Oerlemans, W. Bult, M. Bos, G. Storm, J. W. Nijsen, W. E. Hennink, Pharm. R 27, 2569 (2010)

4. V.P. Torchilin, Pharm. R 24, 1 (2007)

5. Q. Zhou, L. Zhang, T. Yang, H. Wu, Int. M 13, 2925 (2018)

6. M. Ghezzi, S. Pescina, C. Padula, P. Santi, E. Del Favero, L. Cantù, S. Nicoli, J. C. R 332, 313 (2021)

7. M. Amin, A. M. Butt, M. W. Amjad, P. Kesharwani, Nanotechnology-Based Approaches for Targeting and Delivery of Drugs and Genes, 168 (2017)

8. K. Matyjaszewski, J. Xia, Chem. Rev. 101, 2922 (2001)

9. J. Wang, K. Matyjaszewski, J. Am. Chem. Soc 117, 5614 (1995)

10. K. Matyjaszewski, Macro. M 45, 4016 (2012)

11. B. S. Lokitz, A. W. York, J. E. Stempka, N. D. Treat, Y. Li, W. L. Jarrett, C. L. McCormick, Macro.M 40, 6473 (2007)

12. S. Perrier, Macro.M 50, 7434 (2017)

13. N. Rapoport, Pro. Poly. Sci. 32, 964 (2007)

14. H.Choudhury, P. Manisha, B. Gorain, B. Chatterjee, T. Madheswaran, S. Md, K.K. Mak, M. Tambuwala, M.K. Chourasis, P. Kesharwani, NETLC, 218(2019)

15. C.J.F. Rijcken, O. Soga, W.E. Hennink, C.F. van Nostrum, J. CR 3, 136(2007)

16. I. F. Tannock, D. Rotin, PCR 49, 4373 (1989)

17. A.E. Felber, M.H. Dufresne, J.C. Leroux, ADDR 64, 985 (2012)

18. M.c. Jones, M. Ranger, J.C Leroux, Bio Chem 14, 775 (2003)

19. Y.J. Lee, H.C. Kang, J. Hu, J.W. Nichols, Y. S. Jeon, Y.H. Bae, Biomacromolecule 13, 2946 (2012)

20. H. Lee, J. Pietrasik, K. Matyjaszewski, Macro. M 39, 3914 (2006)

21. J. Jiang, X. Tong, Y. Zhao, J. Am. Chem. Soc. 127, $8290(2005)$

22. B. Yan, J. Boyer, N. R. Branda, Y. Zhao, J. Am. Chem. Soc. 133, 19715 (2011)

23. B. Li, Y. Wu, Y. Wang, M. Zhang, H. Chen, J. Li, R. Liu, Y. Ding, A. Hu, ACS Appl. Mater. Interfaces 11, 8897 (2019)

24. S. V. Aathimanikandan, E. N. Savariar, S. Thayumanavan, J. Am. Chem. Soc. 127, 14922 (2005)

25. O. Jung, Soft Matter 7, 5096 (2011)

26. J. Akimoto, M. Nakayama, K. Sakai, T. Okano, Mol. Pharm. 7, 928 (2010)

27. F. Meng, W. E. Hennink, Z. Zhong, Bio. 30, 2180 (2009)

28. M. Huo, Y. Liu, L. Wang, T. Yin, C. Qin, Y. Xiao, L Yin, J Liu, J. Zhou, Mol. Pharm 13, 1751 (2016)

29. C. Liu, J. Yuan, X. Luo, M. Chen, Z. Chen, Y. Zhao, X. Li, Mol. Pharm 11, 4260 (2014) 